\title{
Role of preventive national program in combating diabetes mellitus and cardiovascular diseases
}

\author{
Osman El-Labban* \\ Family Medicine Consultant \& head of Department, Al Zahra Hospital, Dubai, UAE
}

\section{Introduction}

\section{Shining a light and sounding an alarm}

According to a recent editorial in Lancet journal "Type 2 diabetes is becoming the plague of the 21 st century" and "threatens to reduce life expectancy for future generations globally" [1].

Diabetes is a global public health crisis which was fueled by rapid urbanization, nutrition transition, and increasingly sedentary lifestyles.

I think everybody agrees with me on the magnitude of this worldwide epidemic of diabetes which is invading almost every country in the world and spreading among adults \& pregnant women. The great magnitude of type 2 diabetes is not restricted to adults but involve children. This implies on us to handle this crisis through multiple approaches rather than concentrating on one aspect of management.

Let me summaries my suggested different approaches which we have to address in dealing with diabetes mellitus crisis as follows:

\section{Prevention}

Upon trying to approach such a problem, we should be far-sighted and think deeply in the problem and recognize that the current numbers of diabetic patients represent only the numbers in the tip of the iceberg of the true magnitude of the problem, while the bigger portion of the iceberg is hidden below the water and is represented by a larger number of high risk patients and pre-diabetes patients. And with time, the tip of the iceberg would increase in size through adding more and more diabetic patients secondary to an annual 5-10 percent progress of pre-diabetic to diabetic cases.

Healthcare services budgets of different governments had been depleted due to the vicious circle of increase in the number of diabetic patients necessitating an increase in the budget of medical services. Prevention is a vital investment as diabetes threatens the economics of every nation and retards it.

Another good reason for adopting prevention approach is the escalating tendency of the cost healthcare services due to the complexity of treatment of the problem, from those patients controlled on diet alone up to oral hypoglycemic agents and reaching to insulin injections.

In addition to above reasons, the presence of microvascular and macro-vascular complications in diabetes come to add more and more costs on the healthcare services. It is well known that diabetic patients carry the worse prognosis of acute coronary syndrome and diabetes is the main etiology for chronic renal failure.

Primary prevention is what should we aim to and which can take two different forms, addressing either the high risk groups of patients whom can easily progress into pre-diabetes stage and later on to diabetes stage or through addressing the whole population trying to change their lifestyle.

\section{Adoption of preventive National program by stakeholders}

We should work hard together on convincing stakeholder in any community to adopt plans and create new public policies seeking to create a National program for prevention diabetes augmented by a true public-private partnership. A detailed National program will be described in the article.

\section{Combating obesity}

Diabetes has grown in parallel with the worldwide rise in obesity which becomes the main risk to develop type 2. Reasons of increasing obesity nowadays, include excessive caloric intake by consuming higher dietary glycemic load and increased sedentary life through long hours of TV watching, long hours of sitting at work and increased mechanization and driving hours.

Obesity \& especially abdominal obesity is associated by a state of hyperinsulinemia secondary to insulin resistance and is the cornerstone element in developing of the metabolic syndrome.

Efforts should put to recommend better ways to lose weight whether by reviewing recommended dietary advices, suggesting ways on how we can incorporate exercises in our daily routine life or by using of new modalities such as acupuncture. Health education on the glycemic index and the identification of the caloric value by reading of food label is of paramount importance in reducing obesity.

In one of my blogs, I proposed the idea of creating a healthy fast-food which serves the commercial purpose of most fast-food companies, but which is less hazardous on the general health of the population.

Public health strategies should target the obesogenic environment that amplifies the optimal triggers for susceptible individuals.

Reducing the prevalence of obesity would reduce the risk of developing diabetes as well as many other chronic diseases related to lifestyle (Figure 1).

Correspondence to: Dr. Osman El-Labban, Family Medicine Consultant \& head of department, Al Zahra Hospital Dubai, UAE; E-mail: labban53@hotmail.com

Received: April 22, 2016; Accepted: May 21, 2016; Published: May 24, 2016 


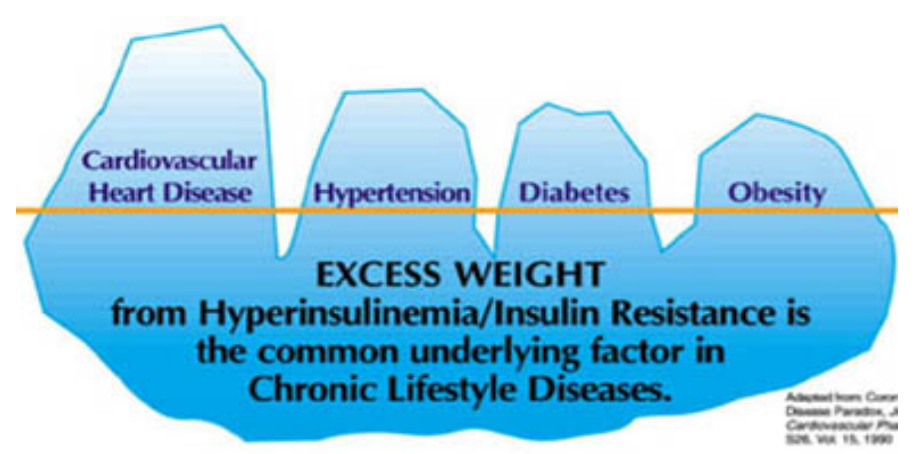

Figure 1. Reducing the prevalence of obesity would reduce the risk of developing diabetes as well as many other chronic diseases related to lifestyle.

\section{Need for more advanced research}

Research is requested in various directions whether to

$\checkmark \quad$ Identify the congenital element of the disease as type 2 diabetes being a product of the interplay between genetic and environmental factors

\section{$\checkmark \quad$ Have better understanding of the pathology of the disease}

$\checkmark \quad$ Searching for new modalities of treatment and methods of delivering insulin to type 1 and type 2 patients whether by the use of insulin pumps or by giving it orally or nasally.

$\checkmark \quad$ Searching for ways of regeneration, activation and stimulation of pancreatic cells hoping to enhance insulin release. And in this issue, we need more researches on the use of stem cells to regenerate pancreatic cells.

\section{Empower patients \& improve ways to deliver health education}

We have to seek to understand the psychology of diabetic patients and offer better ways to educate them on the importance of self-care as well involving them as the main players in the process of management of their diabetes rather than imposing on them instructions and orders.

The ideal settings would be to motivate patients through providing counseling on their diabetes status and health-educate them to the level that permits them to take their own correct decisions. A multidisciplinary team approach is needed in this setting which would include nurse case-manager, health educator, nutritionist and pharmacist in addition to physiotherapist and occupational therapist whenever need.

\section{Smart use of IT and social media}

Healthcare care system in the 21 st century is expected to make use of IT technology more efficiently to:

- Create electronic health record

- Create registry for diabetic cases

- Draw epidemiology data \& statistics

- Link healthcare provider and patient electronically

- Use of smart mobile Applications in promoting of healthcare services

In the same line, social media nowadays plays an important role in our social life and can be employed to promote:

- Public health awareness
- Health education on diabetes

- Promotion of diabetes self-management concepts

- Networking among patients and healthcare providers

\section{Magnitude of the burden of the problem}

Chronic diseases retard economic growth.

According to a research article published in BMC Public Heath in 2010, "Assessment of the Direct Medical Costs of Diabetes Mellitus and it's complications in the UAE", Diabetes mellitus is a major health problem in UAE.

The aim of the study was to estimate the direct annual cost of Diabetes Mellitus and its related complications among patients in $\mathrm{Al}$ Ain City, UAE. As it is clear Diabetes Mellitus direct treatment costs increase in the presence of related complications and in case of progression of the disease (Figure 2). To reduce the impact on healthcare resources, efforts should be made to prevent progression of DM complications by implementing guidelines for diabetic care, screening for complications and better management.

The same study showed that a diabetic case controlled on diet only and without complications costs around 1,605 USD, while diabetic patient who is in insulin costs around 7,086 USD. A diabetic patient with hypertension has an annual cost of 6,210 USD. Hospitals costs constituted the largest proportion of overall treatment costs.

There had been some other studies regarding the total costs. According to the International Diabetes Federation (IDF) in 2010, expenditure is $11.6 \%$ of total healthcare expenditure. In USA, American Diabetes Association showed an annual cost exceeded 174 billion USD in 2007. In Italy, direct cost per DM patient was 2,589 Euro compared to 1,682 Euro for non-diabetic patient in 2006. CODE-2 study done in eight European countries showed 10 million people with type 2 Diabetes with a cost of 29 billion Euro in the year 1999.

The diagram below showed the projections of the number of people between 20-79 years old with Diabetes in the year 2013-2035(Figure 3).

Annual treatment cost for patients with complications are shown in

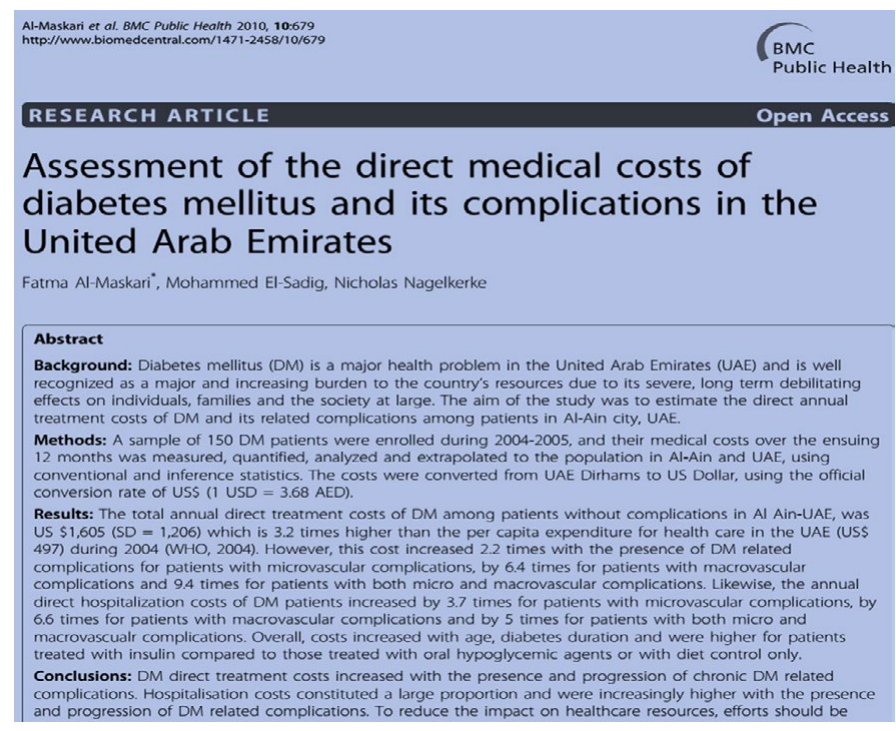

Figure 2. Increase in the presence of related complications and in case of progression of the disease. 


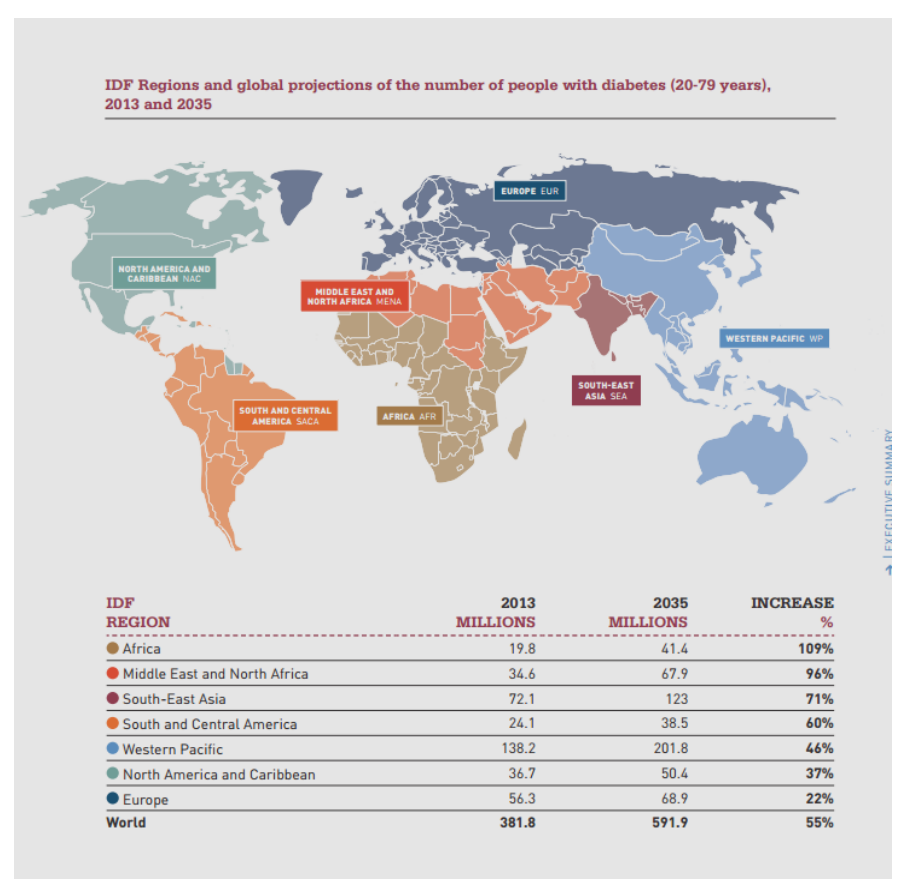

Figure 3. The diagram below showed the projections of the number of people between 20 79 years old with Diabetes in the year 2013-2035.

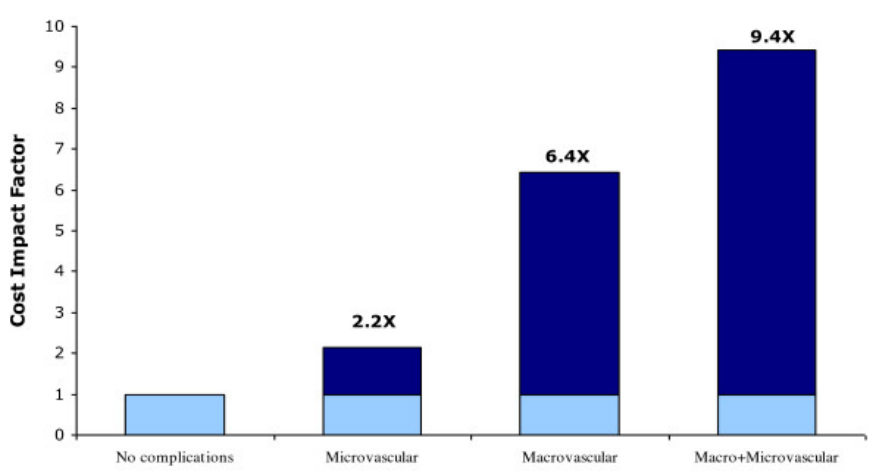

Figure 4. Annual treatment cost for patients with complications are shown in the graph below as magnitude of multiple of cost of diabetic patient without complications.

the graph below (Figure 4) as magnitude of multiple of cost of diabetic patient without complications.

"An ounce of prevention is worth a pound of cure"

\section{Diabetes is preventable and controllable}

There are few questions asked relating to prevention which we need to answer before proceeding in discussing my suggested preventable National program:

- Why do we need prevention?

- Why are prevention program hard?

- Who implements prevention programs?

- What are the levels of prevention programs?

- What is the evidence of benefit in prevention programs?

- How can primary prevention program be implemented in any country?

\section{Why do we need prevention}

Around $5-10 \%$ of people with pre-diabetes become diabetic yearly. The diagram below explains the continuous flow of newly diagnosed cases which jeopardize the budget in any country (Figure 5).

\section{Why are prevention programs hard}

- "Hard to sell" to people as success is marked by non-event result.

- "Hard to implement" as reduction of risk needs individual behavior changes

- "Hard to finance" as costs are immediate \& benefits take a longer time to appear

\section{Who implements prevention programs}

Prevention is a prime function of a family physician coordinated in a multidisciplinary team approach. Family physician drives the health of the community and seeks cost-saving and cost-effective community projects.

\section{What are the levels of prevention programs}

This is best shown in the diagram below (Figure 6)

Primary prevention aims in preventing the development of risk factors leading to diabetes in susceptible populations or individuals. Risk factors that predict the progression are obesity, elevated fasting, 2-hour blood glucose and fasting insulin concentrations. There are two approaches in primary prevention: high-risk approach and community-based approach.

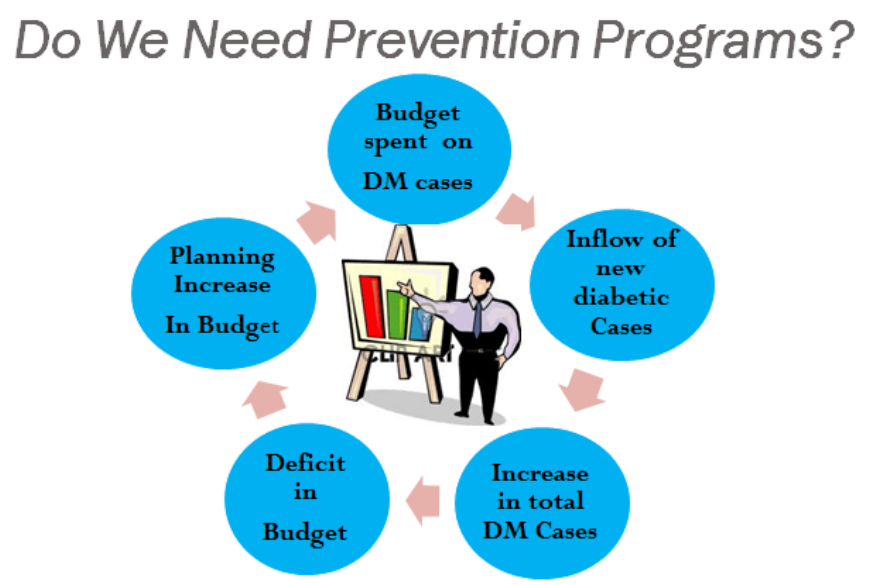

Figure 5. The diagram below explains the continuous flow of newly diagnosed cases which jeopardize the budget in any country.

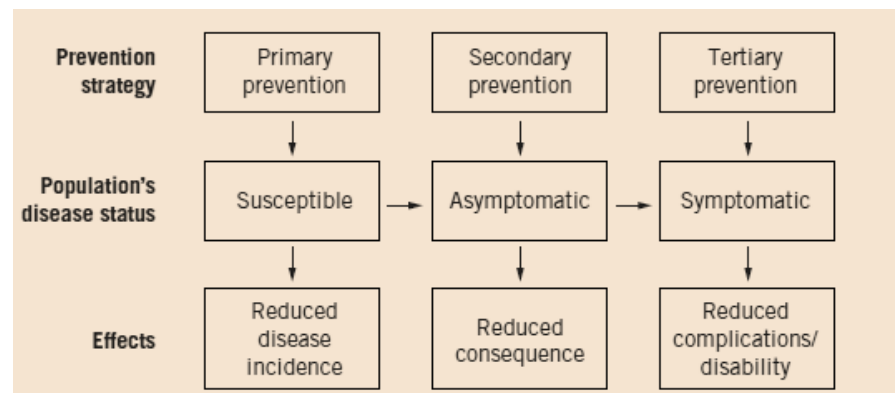

Figure 6. Levels of prevention programs. 
High-risk approach focuses on individuals at highest risk of diabetes. It empowers these patient with pre-diabetes to take charge of their health and well-being. Patients learn ways to incorporate healthier eating and moderate physical activity, as well as problem-solving, stress reduction and coping skills into their daily lives.

These includes individuals 45 years of age and older, overweight, have a family history of type 2 diabetes, high blood pressure, physically active fewer than 3 times a week, gestational diabetes or gave birth to a baby who weighed more than 9 pounds and with lab test showing HBA1C $5.7-6.4 \%$ or FPG $100-125 \mathrm{mg} / \mathrm{dL}$ or OGTT $140-199 \mathrm{mg} / \mathrm{dL}$.

Community-based approach considers all determinants of health enabling people to increase control of their lifestyle. In this approach, one would think of multi-sectors \& multi-strategies. It encourages physical activity, healthy eating and healthy weight for the entire population. It promotes healthy life styles for all people of all ages within the community and looks for small changes made by majority. Thus it reduces risk factors and makes healthy choices easier choices. But, it requires new health policies or actions by stakeholders.

Secondary prevention is early diagnosis and effective control of diabetes to avoid or at least delay the progress of the disease.

Tertiary prevention lastly, includes measures taken to prevent complications and disabilities due to diabetes.

Prevention is a vital investment and attack more than one disease (Figure 7).

Investment in prevention of diabetes including fighting of obesity and avoiding of sedentary life and consuming healthy food would bring returns in prevention of cardiovascular diseases too, as both diseases share many common risk factors

\section{What is the evidence of the benefit of prevention}

Multiple studies on high riskgroups rather than general populations, had given the evidence for the value of primary prevention in reversing of progression from pre-diabetes to diabetes stage or at least slowing the process of this progression knowing that people with IGT has a 2-7 fold higher risk of progression to Type 2 diabetes than persons with normal glucose tolerance.

Reviewing randomized controlled trials below shows the feasibility and benefit of various strategies to prevent or delay the onset of type 2 diabetes among pre-diabetes patients:

- Finish study

- Diabetes Prevention Program

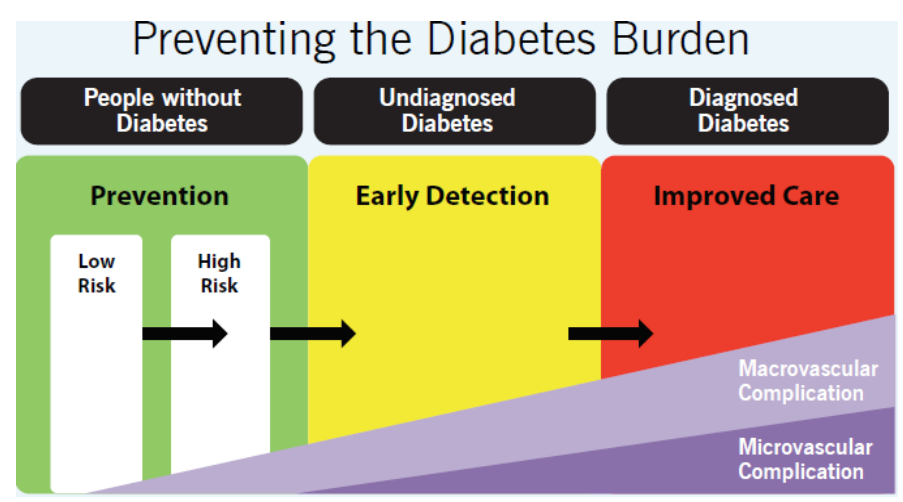

Figure 7. Prevention is a vital investment and attack more than one disease.
- Troglitazone in Prevention of Diabetes (TRIPOD)

- STOP-NIDDM trial

- The Da Qing Chinese study

Finish study: Subjects with IGT were randomized to receive either brief diet and exercise counseling (control group) or intensive individualized instruction on weight reduction, food intake, and guidance on increasing physical activity (intervention group).

After an average follow-up of 3.2 years, there was a $58 \%$ relative reduction in the incidence of diabetes in the intervention group compared with the control subjects.

Diabetes prevention program: Subjects were randomized to one of 3 intervention groups, intensive nutrition \& exercise counseling ("lifestyle") group or either of two masked medication treatment groups: metformin group or placebo group 2.8 years. here was $58 \%$ relative reduction in the progression to diabetes in the lifestyle group (absolute incidence 4.8\%), 31\% relative reduction in the progression of diabetes was observed in the metformin group (absolute incidence 7.8\%) compared with Control subjects (absolute incidence 11.0\%).

Troglitazone in prevention of diabetes (TRIPOD) study: Randomized to receive either placebo or troglitazone.

After a median follow up of 30 months, the annual incidence of type 2 diabetes in the two groups was 12.3 and $5.4 \%$, respectively

STOP-NIDDM trial: Randomized in a double-blind fashion to receive either the glucosidase inhibitor acarbose or a placebo. After 3.3 years, a $25 \%$ relative risk reduction in progression to diabetes, based on one OGTT, was observed in the acarbose treated group compared with the placebo group

The Da Qing Chinese study: Showed that over 6 years there were significant and similar reductions in the incidence of diabetes in subjects with IGT who were randomized to diet, exercise, or combined diet-exercise treatment groups.

\section{How can primary prevention national program be implemented in any country}

Upon the recommendations of the ministry of Health, the cabinet takes the initiative to adopt a National Program to combat Diabetes and Cardiovascular diseases. Cabinet acts as stakeholders through recognition and adoption of the program, nomination of specialized national committee for Diabetes Prevention, legislation of new policies and laws, and establishing food security policies such as subsidizing the cost of healthy food.

\section{Below is a distribution of roles among ministries (Figure 8)}

Ministry of Health sets the role of the national committee, prepares the plans and new policies, sets mass catering recommendations and policies, empowers primary health care (PHC) and ensure availability of physical activity trainer and dietician in PHC.

Ministry of Health supports planning \& backing up of periodic examination, screening programs $\&$ health promotion with emphasis on proper training of medical students on most common chronic diseases of the country. Ministry helps in arranging workshops, conferences \& public awareness campaigns

National Steering Committee consists of medical professionals in endocrinology, diabetology and cardiology in addition to experts in the 


\section{Cabinet acting as Stakeholders}

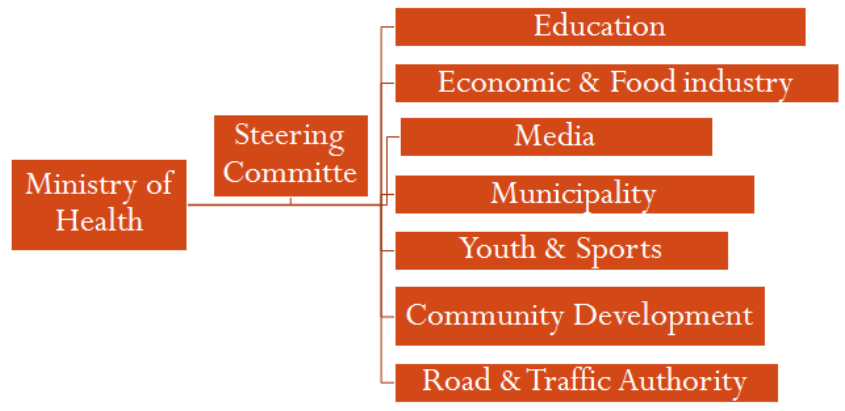

Figure 8. Below is a distribution of roles among ministries as seen in the picture.

field of prevention of Diabetes Mellitus and Cardiovascular diseases.

National steering committee outlines the interventions and implementation plans set by the Ministry of Health. The committee coordinates the work of other ministries and promotes national mass catering policies in food industry. Committee also organizes and performs research and evaluation processes of the public policy effectiveness.

The role of the ministry of education includes adding healthy eating, physical activities and diabetes education in school curriculum. Ministry of education also implements an increase in sports hours, ensures availability of sports facilities and availability of healthy foods in cafeterias and vending machines. The ministry of education creates nutritional policy banning high fat and high sugar snack foods in the schools. Ministry of education helps young people to learn how to cook nutritious, healthy and low fat foods. Lastly, they also health educate the parents about what their children are learning in school.

The role of the Ministry of Economics and food industry includes observing mass catering policies in food industry like reducing saturated fat content and salt content of manufactured food through obligation and accompanying health-educational campaigns. Ministry also increases availability and helps in reducing costs of the healthy foods and promotes healthy food choices and limits the aggressive marketing of unhealthy food to children. They enforce sticking of nutrition \& energy content labeling for all packaged food including fast food.

Ministry of economic uses tax policies to encourage the consumption of healthier foods and puts taxes on cigarettes and pumps collected tax in health projects.

Ministry enforces the adoption of health insurance companies the strategy of accepting of the periodic examination \& screening tests in health insurance policies for insured clients.

The role of Ministry of Youth and Sports helps in creating the spirit of sports adoration among youth and in providing access to community recreational facilities. Ministry will arrange summer camps and will observe sports sponsors of activities. Ministry will train volunteers who aim to promote population health.

The role of the Ministry of Media is to adopt WHO health awareness calendar with creation and plotting of health programs in the TV maps. Live healthy cooking shows for National cuisines would allow people to cook popular plates in a healthier way. Ministry of media helps in health literacy of the community and promoting educational campaigns on how to read and understand food labels.

Ministry develops local appropriate health messages related to diet, physical activity and weight control in radio and television. And also helps in the proper use of social media to promote healthy lifestyle. Ministry also would embed health-promoting messages and storylines into the entertainment and news programs to eliminate messages that counter health messages.

The role of the municipality is to inspect restaurants checking whether they are abiding low-salt, low fat and no added sugars policies in food preparation. Municipality also enforces replacing dietary Trans Fat with polyunsaturated fat. Municipality also would ensure the availability of a wide range of healthy foods and drinks without sugar, in competitive prices in addition to the availability of non-smoking areas in restaurants.

The role of the Community development ministry is to adopt the partnership between government and non-governmental organizations. It creates the individual's internal sense of responsibility in choosing healthy lifestyle. It also helps in forming and supporting networks of community-based organizations committed to improve nutrition and physical activities.

Lastly, the road and traffic authority adopts policies that promote walker-friendly communities and encourage walking and the use of bicycle by providing bike lanes and secure bicycle parking. The road and traffic authority also encourages families to use of public transportation by having discounts on fares for families over the weekend.

In summary, prevention is a vital investment. There is a proverb that says, "Prevention is better than cure." I believe that it is better to avoid problems in the primarily rather than trying to fix problems once it arise. There is a need for comprehensive health reform legislation and solid infrastructure of community-based programs.

\section{References}

1. Al-Maskari F, El-Sadig M, Nagelkerke N (2010) Assessment of the direct medical costs of diabetes mellitus and its complications in the United Arab Emirates. BMC Public Health 10: 679. [Crossref]

Copyright: (C2016 El-Labban O. This is an open-access article distributed under the terms of the Creative Commons Attribution License, which permits unrestricted use, distribution, and reproduction in any medium, provided the original author and source are credited. 